\title{
FLOTAC and Mini-FLOTAC for uro-microscopic diagnosis of Capillaria plica (syn. Pearsonema plica) in dogs
}

\author{
Maria Paola Maurelli ${ }^{1}$, Laura Rinaldi $i^{*}$, Giuseppe Rubino ${ }^{2}$, Riccardo Lia ${ }^{3}$, Vincenzo Musella ${ }^{4}$ \\ and Giuseppe Cringoli ${ }^{1}$
}

\begin{abstract}
Background: Capillaria plica (syn. Pearsonema plica) is a nematode that resides in the urinary bladder and rarely in ureters or in the kidney pelvis of various carnivores, especially foxes and dogs. Urine sedimentation technique is actually the only diagnostic tool that permits the identification of C. plica eggs, but its sensitivity is low and when an infection is suspected (or when it is necessary to confirm treatment efficacy) more than one examination of urine sediment should be performed. The present paper reports a clinical case of natural C. plica infection in a dog from southern Italy. In addition, two new techniques, FLOTAC and Mini-FLOTAC, were used for the diagnosis of C. plica in dog urine and compared with the technique of sedimentation.

Results: Using FLOTAC with fresh urine and sodium chloride as flotation solution, were obtained the best results for the diagnosis of $C$. plica in dog urine in term of eggs counted (mean eggs per $10 \mathrm{ml}$ of urine $=70.3$ FLOTAC vs 40.3 Mini FLOTAC vs 32.8 sedimentation) and coefficient of variation (CV\%) (6.2 FLOTAC vs 13.4 Mini-FLOTAC vs 32.9 sedimentation).

Conclusions: The FLOTAC was the more sensitive method, but also the Mini-FLOTAC could be a valid alternative diagnostic method because gave better results than the classical sedimentation and can be used in place of the FLOTAC in laboratories where the centrifugation step cannot be performed.
\end{abstract}

Keywords: Capillaria plica, Dog, FLOTAC, Mini-FLOTAC, Sedimentation technique

\section{Background}

Capillaria plica (Syn. Pearsonema plica) [1], commonly known as the "bladderworm", is a nematode that resides in the urinary bladder and rarely in ureters and kidney pelvis of various carnivores, especially foxes and dogs [2-4]. C. plica has an indirect life cycle and involves an earthworm as the intermediate host. Once the definitive host eats earthworms with the first stage larvae, these moult to the second stage and then to the third stage within the wall of the small intestine. Finally, the larvae reach the bladder, where they moult to adults and embed themselves deep into bladder mucosa [5]. The prepatent period in dogs has been reported to be around

\footnotetext{
*Correspondence: Irinaldi@unina.it

'Department of Veterinary Medicine and Animal Productions, University of Naples Federico II CREMOPAR, Campania Region, Naples, Italy

Full list of author information is available at the end of the article
}

8-10 weeks [6]. C. plica is considered to be of low pathogenic significance and in most cases, the parasite establish only asymptomatic infections [7]. The symptomatic cases described in domestic dogs have showed dysuria, haematuria, pollakiuria, polydipsia and urinary incontinence [8-10]. Recently, C. plica has been also suggested to be a contributing factor in glomerular amyloidosis in dogs [5].

Urine sedimentation technique is actually the only diagnostic tool that permits the identification of $C$. plica eggs [5]. It is a qualitative method and its sensitivity is low and when an infection is suspected (or when it is necessary to confirm treatment efficacy) more than one examination of urine sediment should be performed [11].

The FLOTAC techniques are quantitativce methods described for the copromicroscopic diagnosis of parasites in humans and animals [12] and permit a 
multivalent detection of dog parasites including Crenosoma vulpis [13], Spirocerca lupi [14], Angiostrongylus vasorum [15] and Ancylostoma caninum [16]. Interestingly, FLOTAC has been also successfully utilized for the diagnosis of Schistosoma haematobium in human urine [17].

Mini-FLOTAC is an evolution of FLOTAC conceived to perform multivalent faecal egg counts through an easy approach. It has a simple design, which is based on only two components, the base and the reading disc. The device includes also two 1-ml flotation chambers designed for optimal examination of faecal sample suspensions (total volume $=2 \mathrm{ml}$ ) [18]).

Mini-FLOTAC has been already validated for the diagnosis of most important human nematodes (e.g. soiltransmitted helminths) and trematodes (e.g. Schistosoma) [19] and showed promising results in veterinary parasitology for the diagnosis of helminths (e.g. ascarids, hookworms, trichurids, gastro-intestinal nematodes and liver flukes) in pets and livestock [20].

The aims of this work are: 1) to evaluate two new quantitative techniques, FLOTAC and Mini-FLOTAC, for the diagnosis of $C$. plica in dog urine; 2) to compare these two innovative techniques with the technique of sedimentation, in terms of sensitivity and precision.

\section{Results}

The Figure 1 shows the mean C. plica eggs per $10 \mathrm{ml}$ of dog urine calculated for each of two flotation solutions (FSs): sodium chloride (FS2) and zinc sulphate (FS7) and for two preservation methods: fresh and fixed urine, along with the coefficient of variation $(\mathrm{CV})$, using FLOTAC.
C. plica eggs were detected with both FSs either using fresh urine and fixed urine. However, the highest count (26.0 eggs per $10 \mathrm{ml}$ ) and lowest CV (3.8\%) were obtained with fresh urine $(\mathrm{P}=0.04)$ and using FS2 $(\mathrm{P}=0.04)$ i.e., sodium chloride.

All the three techniques (FLOTAC, Mini-FLOTAC and urine sedimentation) were capable to detect C. plica eggs. For aliquot 3 , the mean number of $C$. plica detected with FLOTAC was significantly higher than those detected by Mini-FLOTAC and sedimentation (40.7 eggs per $10 \mathrm{ml}$ of urine vs 28.3 and 20.7, respectively); in addition the CV\% detected with FLOTAC was lower than the values detected by the other techniques (5.5 vs 12.8 and 36.0, respectively). Also for the aliquot 4, FLOTAC gave higher mean of $C$. plica detected (99.8 eggs per $10 \mathrm{ml}$ of urine $v s 52.3$ and 44.8, respectively) and lower CV\% (6.8 vs 14.0 and 29.7 respectively) than the other two techniques (Figure 2).

\section{Discussion}

Reports of C. plica infection in dogs and cats are limited and the actual prevalence could be underestimated. In Italy only one clinical case in a hunting jagd terrier in Parma [5] and one in an European cat from Pisa [21] have been reported so far. Many studies, however, have revealed a high prevalence in red foxes in several European countries, with prevalence ranging from 50\% to $80 \%$ [22]. Thus, fox populations are likely the main source of infection for hunting dogs [5].

The dog examined in our present study was born in Spain where $C$. plica is widespread in foxes [23] and lived in the Apulia Region for less of one year. None of other dogs that lived together presented the same symptomatology.

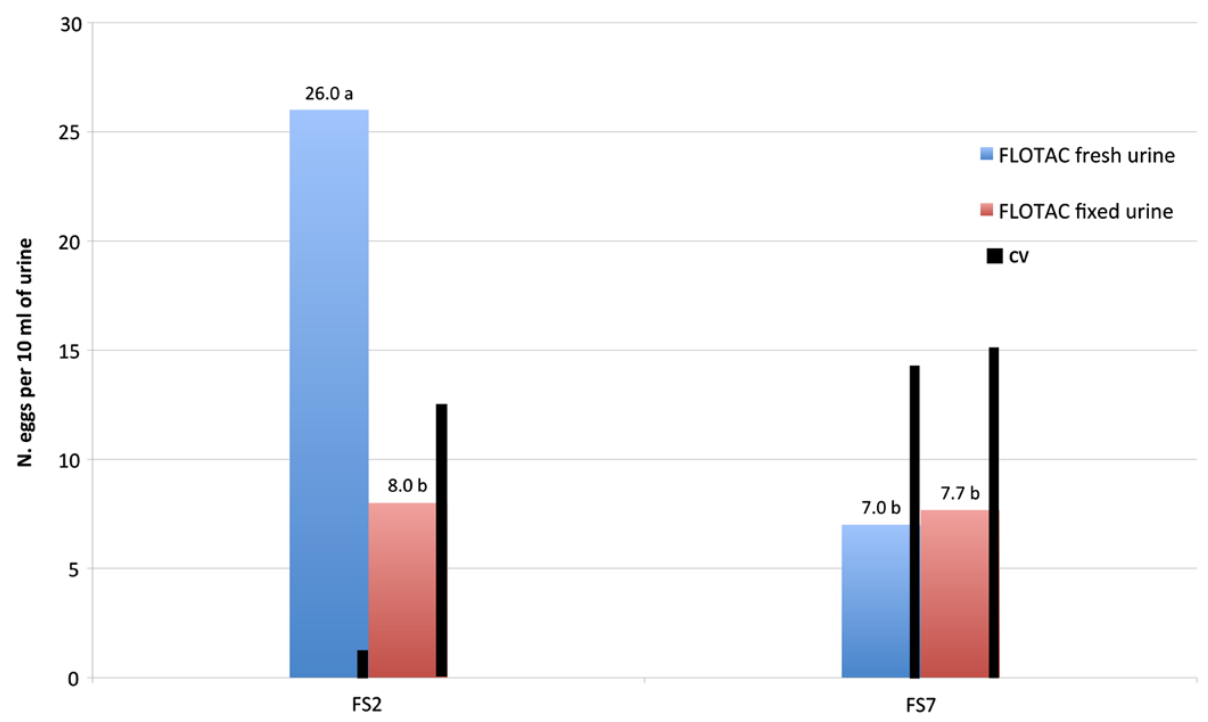

Figure 1 Mean C. plica eggs and coefficient of variation (CV\%) detected by FLOTAC using two flotation solutions (FS2 and FS7) on fresh and fixed urine. Significant differences $(P<0.05)$ for different letters $(a, b)$. 


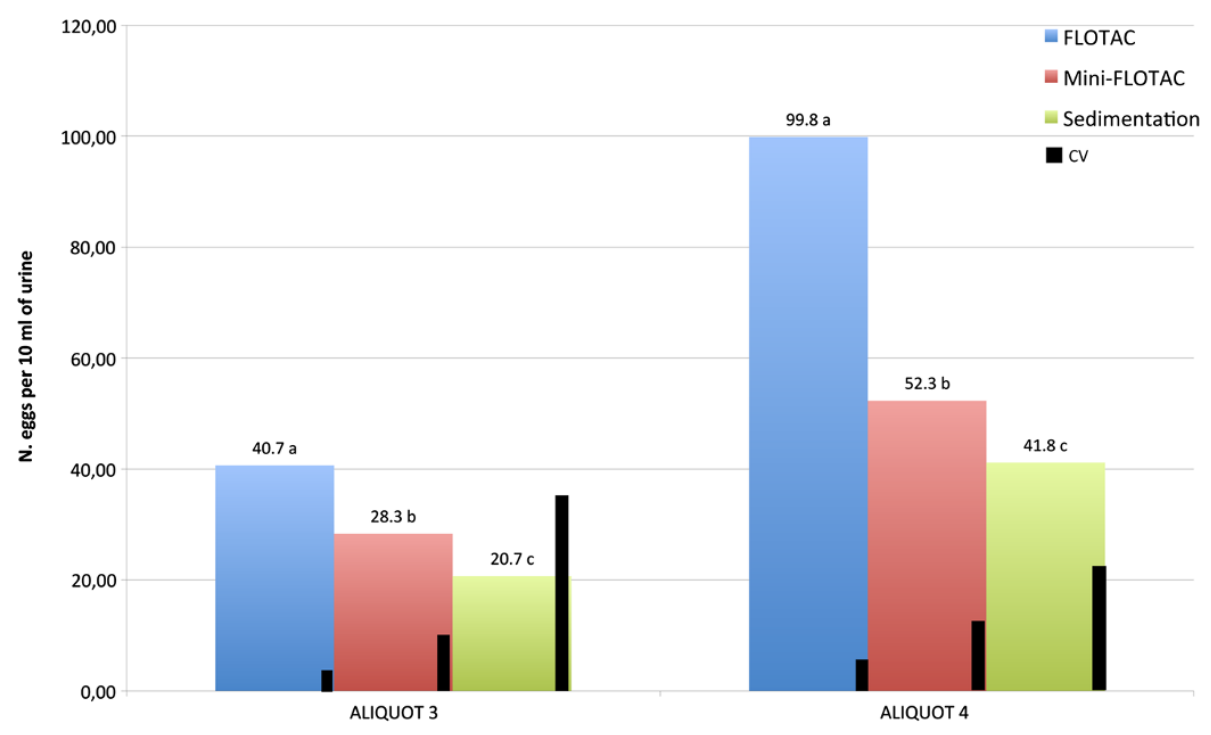

Figure 2 Mean C. plica eggs and coefficient of variation (CV\%) detected by FLOTAC, Mini-FLOTAC and urine sedimentation techniques. Two aliquots (aliquot 3 and 4) of fresh urine were used. FS2 solution was used for FLOTAC and Mini-FLOTAC. Significant differences $(P<0.05)$ for different letters $(a, b, c)$.

The treatment with febendazole at a dose rate of $50 \mathrm{mg} / \mathrm{kg} /$ day for 10 days was successfully used, as reported also in other cases in literature $[6,21,24]$.

\section{Conclusions}

The results of this work provide important new information on the performance of available methods for the intra vitam diagnosis of C. plica in dogs. The findings have suggested that the FLOTAC, using fresh urine and sodium chloride (FS2), may improve the ability to accurately diagnose capillariosis in dog urine and permit to overcome the limits of the "classical" sedimentation method. The FLOTAC has yet used successfully also for the diagnosis of S.haematobium in human [17], and so could be proposed as good method for diagnosis of parasites in urine. An alternative diagnostic method is MiniFLOTAC that gave better results than the classical urine sedimentation technique and can be used in place of the FLOTAC in laboratories where the centrifugation step cannot be performed. However, further studies are required to confirm the findings of the present case report.

\section{Methods}

\section{Case report}

A 4-year-old Labrador Retriever, male, born in Spain and living in the Apulia Region (southern Italy) was presented to the referring clinician with a recurrence of dermatitis at flat thighs, abdomen and testicles. The dog was treated previously with cortisone and fluid therapy with no positive outcomes. Clinical diagnosis showed palpable abdomen, internal organs evaluable and a productive cough. The dog was regularly vaccinated and treated against ectoparasites.
Chest $\mathrm{x}$-ray examination revealed not altered structures. Abdominal ultrasound revealed chronic cystitis with thickening of the bladder wall with irregular margins. A serum chemical profile and complete blood count $(\mathrm{CBC})$ revealed eosinophilia, whereas other parameters were within normal limits.

A macroscopic examination of urine revealed haematuria, while a microscopic examination of urinary sediment revealed the presence of several $C$. plica eggs. The dog was treated with febendazole at a dose rate of $50 \mathrm{mg} / \mathrm{kg} /$ day for 10 days and showed a rapid recovery, with resolution of all clinical signs.

\section{Flotation solutions choice for FLOTAC and Mini-FLOTAC and urine preservation}

To determine the optimum flotation solution for FLOTAC and Mini-FLOTAC and the possibility to preserve urine, two FSs: sodium chloride (FS2), specific gravity (s.g. 1200) and zinc sulphate (FS7) (s.g. 1350) were compared using either fresh urine and urine fixed with $5 \%$ formalin (because could be a good alternative method of preservation of urine from collection until analysis).

For this aim one hundred and twenty $\mathrm{ml}$ of urine were collected from the C. plica infected dog (see Case report paragraph).

The urine was divided in two aliquots (Aliquot 1 and Aliquot 2) of $60 \mathrm{ml}$ each: Aliquot 1 was analyzed as fresh, Aliquot 2 was fixed with $3 \mathrm{ml}$ of a $37 \%$ formaldehyde solution and analyzed after 7 days. Each of two aliquots was carefully homogenized and 6 tubes were filled with $10 \mathrm{ml}$ of urine, to have 3 replicates for each of the two FSs used. 
The tubes were centrifuged at $240 \mathrm{~g}$ for 5 minutes and the supernatant poured off and discarded. The tube with the remaining pellet was then filled with each FS until $11 \mathrm{ml}$ level mark and slowly agitated.

The suspension was transferred into the two chambers of a FLOTAC apparatus using a disposable pipette. The apparatus was centrifuged at $120 \mathrm{~g}$ for $5 \mathrm{~min}$, and translated to separate the floating eggs from the urinary debris. All eggs visible through the ruled window of the reading disc were counted under a microscope at $100 \times$ total magnification.

\section{Comparison of FLOTAC, Mini-FLOTAC and urine sedimen- tation techniques}

The following three techniques were compared for the diagnosis of C. plica in dog urine: the FLOTAC basic technique [12,17], the Mini-FLOTAC technique [18] and the urine sedimentation technique [25].

Fresh urine and FS2 (for FLOTAC and Mini-FLOTAC) were used based on the results obtained from the previous experiment (see Flotation solutions choice for FLOTAC and Mini-FLOTAC and urine preservation paragraph).

Two aliquots (Aliquot 3 and Aliquot 4) of $200 \mathrm{ml}$ each of urine were collected from the same dog naturally infected by C. plica. Each aliquot of urine was accurately homogenized and divided in 18 tubes each filled with $10 \mathrm{ml}$ of urine, to have 6 replicates for each of the three diagnostic method. The tubes were randomly assigned to the three techniques.

For the urine sedimentation technique, the tubes were left to room temperature for $1 \mathrm{~h}$, the supernatant was poured off [25] and the tubes were centrifuged at $2000 \mathrm{~g}$ for 2 minutes. After the supernatant was discarded, the pellet has been examined on a glass slide.

For the FLOTAC and Mini-FLOTAC techniques, the tubes were centrifuged at $240 \mathrm{~g}$ for 5 minutes, the supernatant was poured off and $6 \mathrm{ml}$ of FS2 were added to tubes to fill the FLOTAC chambers, whereas $2 \mathrm{ml}$ of FS2 were added to tubes to fill the Mini-FLOTAC chambers (for both techniques the entire pellet was examined).

For each diagnostic technique, C. plica eggs were counted for all replicates using a light microscope at $10 \times$ total magnification. The analytic sensitivity of all the three techniques was 1 egg per $10 \mathrm{ml}$ of urine.

\section{Statistical analysis}

The arithmetic mean eggs per $10 \mathrm{ml}$ of urine, standard deviation $(\mathrm{SD})$, and coefficient of variation $(\mathrm{CV})$ were calculated for the two FSs, preservation method and diagnostic technique. Differences between FSs were analyzed using one-way ANOVA with post hoc Fisher's least significant difference (LSD). All statistical analyses were carried out using STATA version 10.0 (Stata Corp.; Texas, USA). In addition, a likelihood ratio test of the equality of the $\mathrm{CV}$ of normally distributed populations was performed using software developed by the Statistical Services at the Forest Products Laboratory (USA; http://www1.fpl.fs.fed.us/covtestk.html).

\section{Consent}

Written informed consent was obtained from the owner of dog to publish data and information in this report.

\section{Competing interest}

GC is the inventor of the FLOTAC and Mini-FLOTAC apparatus. In case the currently ongoing developments and validations of these apparatus are successful, the methods will be licensed free of charge to the World Health Organization and interested public non-commercial research centers. All other authors have no conflicts of interest.

\section{Authors' contributions}

MPM and LR analyzed the results and drafted the manuscript. MPM performed the microscopic analyses. VM performed the statistical analyses. GR and RL performed the clinical study and parteciped in the design of study. GC performed the study design and sampling, helped to draft the manuscript and revised it. All authors read and approved the final manuscript.

\section{Author details}

'Department of Veterinary Medicine and Animal Productions, University of Naples Federico II CREMOPAR, Campania Region, Naples, Italy. ²Department of Animal Production, University of Bari "Aldo Moro", Valenzano, BA, Italy.

'Department of Veterinary Medicine, University of Bari "Aldo Moro",

Valenzano, BA, Italy. ${ }^{4}$ Department of Health Science, University of Catanzaro Magna Graecia, Catanzaro, Italy.

Received: 18 October 2013 Accepted: 12 August 2014 Published: 2 September 2014

\section{References}

1. Rudolphi CA: Entozoorum Synopsis cui Accedunt Mantissa Duplex et índice locupletissimi. Berolini, Germany: Sumtibus Augusti Rücker; 1819. 811 pp.

2. Shimalov W, Shimalov VT: Helminth fauna of the red fox (Vulpes vulpes Linnaeus, 1758) in southern Belarus. Parasitol Res 2003, 89:77-78.

3. Saeed I, Maddox-Hyttel C, Monrad J, Kapel CMO: Helminths of red foxes (Vulpes vulpes) in Denmark. Vet Parasitol 2006, 168:338-341.

4. Bork-Mimm S, Rinder H: High prevalence of Capillaria plica infections in red foxes (Vulpes vulpes) in Southern Germany. Parasitol Res 2011, 108:1063-1067.

5. Callegari D, Kramer L, Cantoni AM, Di Lecce R, Dodi PL, Grandi G: Canine bladderworm (Capillaria plica) infection associated with glomerular amyloidosis. Vet Parasitol 2010, 168:338-341.

6. Ventura-Morales A, Zaragoza-Vera C, Santamaria-Mayo E, Garcia-Herrera RA: Capillaria plica infection in Mexican cat. J Anim Vet Adv 2012, 11(21):3943-3945.

7. Taylor MA, Coop RL, Wal RL: Parasites of Dogs and Cats. In Veterinary Parasitology. 3rd edition. Oxford: Blackwell Publishing Ltd; 2007:429-430.

8. Meadway W, Skelley JF: Capillaria plica infection in a dog. J Am Vet MedAssoc 1961, 139:907-908.

9. Senior DF, Somon GB, Goldshmidt MH, Joyce T, Bovee KC: Capillaria plica infection in dogs. J Am Vet Med Assoc 1980, 176:901-905.

10. Basso W, Spanhauer Z, Arnold S, Deplazes P: Capillaria plica (syn. Pearsonema plica) infection in a dog with chronic pollakuria: challenges in the diagnosis and treatment. Parasitol Int 2014, 63:140-142.

11. Kirkpatrick CE, Nelson GR: Ivermectin treatment of urinary capillariasis in a dog. J Am Vet Med Assoc 1987, 191:701-702.

12. Cringoli G, Rinaldi L, Maurelli MP, Utzinger J: FLOTAC: new multivalent techniques for qualitative and quantitative copromicroscopici diagnosis of parasites in animals and humans. Nat Protoc 2010, 3:503-515.

13. Rinaldi L, Calabria G, Carbone S, Carrella A, Cringoli G: Crenosoma vulpis in dog: first case report in Italy and use of the FLOTAC technique for copromicroscopic diagnosis. Parasitol Res 2007, 101:1681-1684.

14. Traversa D, Avolio S, Modry D, Otranto D, lori R, Aroch I, Cringoli G, Milillo P, Albrechtová Mihalca AD, Lavy E: Copromicroscopic and molecular assays 
for the detection of cancer-causing parasitic nematode Spirocerca lupi. Vet Parasitol 2008, 157:108-116.

15. Schnyder M, Maurelli MP, Morgoglione ME, Kohler L, Deplazes P, Torgerson $P$, Cringoli G, Rinaldi L: Comparison of faecal techniques including FLOTAC for copromicroscopic detection of first stage larvae of Angiostrongylus vasorum. Parasitol Res 2010, 109(1):63-69.

16. Cringoli G, Rinaldi L, Maurelli MP, Morgoglione ME, Musella V, Utzinger J: Ancylostoma caninum: Calibration and comparison of diagnostic accuracy of flotation in tube, McMaster and FLOTAC in faecal samples of dogs. Exp Parasitol 2011, 128:32-37.

17. Coppola MG, Petrullo L, Gualdieri L, Morgoglione ME, Maurelli MP, Rinaldi L, Musella V, Alfano S, Piccoli S, Cringoli G, Atripaldi L, Rossano F: Abstract Book of XXXVIII Congresso Nazionale AMCLI. Rimini (Italy): AMCLI Milano; 2009:103.

18. Cringoli $G$, Rinaldi L, Albonico M, Bergquist R, Utzinger R: Geospatial (s) tools: integration of advanced epidemiological sampling and novel diagnostics. Geospat Health 2013, 7(2):399-404.

19. Barda B, Zepherine H, Rinaldi L, Cringoli G, Burioni R, Clementi M, Albonico M: Mini-FLOTAC and Kato-Katz: helminth eggs watching on the shore of Lake Victoria. Parasit Vectors 2013, 6:220.

20. Cringoli G, Maurelli MP, Pepe P, Alfano S, Pennacchio S, lanniello D, Rinaldi L: Mini-FLOTAC, a new tool for copromicroscopicdiagnosis of common intestinal nematodes in dogs. Parasit Vectors 2014, 7:356.

21. Rossi M, Messina N, Ariti G, Riggio F, Perrucci S: Symptomatic Capillaria plica infection in a young European cat. J Feline Med Surg 2011, 13(10):793-795.

22. Fernandez-Aguilar X, Mattsson R, Maijer T, Osterman-Lind E, Gavier-Widen D: Pearsonema (syn. Capillaria) plica associated cystitis in a Fennoscandian arctic fox (Vulpes lagopus): a case report. Acta Vet Scand 2010, 52:39-42.

23. Segovia JM, Torres J, Miquel J, Llaneza L, Feliu C: Helminths in the wolf, Canis lupus, from north-western Spain. J Helminthol 2001, 75(2):183-192.

24. Van Veen L: Bladder infection with Capillaria plica in a male dog. Tijdschr Diergeneeskd 2002, 127:393-394.

25. WHO: Basic Laboratory Methods in Medical Parasitology. Switzerland: Genevra; 1991.

doi:10.1186/1756-0500-7-591

Cite this article as: Maurelli et al:: FLOTAC and Mini-FLOTAC for uro-microscopic diagnosis of Capillaria plica (syn. Pearsonema plica) in dogs. BMC Research Notes 2014 7:591.

\section{Submit your next manuscript to BioMed Central and take full advantage of:}

- Convenient online submission

- Thorough peer review

- No space constraints or color figure charges

- Immediate publication on acceptance

- Inclusion in PubMed, CAS, Scopus and Google Scholar

- Research which is freely available for redistribution 